\title{
Inhaltsverzeichnis
}

Vorwort $\quad$ IX

\section{Gespräch und Diskurs}

Claus-Artur Scheier

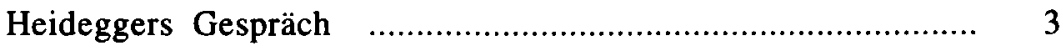

Sigurd Wichter

Kommunikationsebenen und Diskurs

2. Sprache und Literatur

Peter von Polenz

Studentensprache im Duellzwang.

Nach einem wiederaufgetauchten Manuscripte

von Wilhelm v. Polenz (1885) .................................................

Almut Vierhufe

„Bluffende Mimikry“.

Literarische Stil- und Sprachkritik am Beispiel

von Gottfried-Benn-Parodien

Ulla Fix

Die Gedichte satzanfang und Sprachvermögen Sprechenkönnen

Sprichwenndukannst. (Be)greifbare Beziehungen zwischen

Linguistik und Literatur

\section{Matti Luukkainen}

„Das Kunstwahre“ und ,das Naturwahre“.

Zur Semantik im literarischen Text

\section{Sprachkritik und Zeitgeschichte}

Hiroyuki Takada

Kritische Betrachtungen zu Leibniz' Sprachkritik.

Was leistet Leibniz ,betreffend die Ausübung und

Verbesserung" der deutschen Sprache? 
Christine Kaiser

„,...diese armen Worte eines innigen Dankes“.

Zu Fritz Mauthners Danksagung anläßlich seines

siebzigsten Geburtstags

Hans Peter Althaus

„Schleeschaak“. Ein Tenor im Visier von Karl Kraus

Heidrun Kämper

LQI - Sprache des Vierten Reichs.

Victor Klemperers Erkundungen zum Nachkriegsdeutsch

Siegfried Grosse

Politisches Zeitgeschehen und die deutsche Gegenwartssprache

\section{Lexikologie und Lexikographie}

Dieter Stellmacher

Niederdeutsche Schimpfwörterbücher

Klaus-Dieter Ludwig

Pragmatische Markierungen im „Paul“

Hartmut Schmidt

Das Reich der Freiheit

Jörg Kilian

Die Ordnung der Wörter in den Köpfen der Sprecher.

Zum Terminus Gruppe in Hermann Pauls Sprachtheorie

Peter Braun

Augenblicks- und Gelegenheitsbildungen:

für eine begriffliche Differenzierung

Horst Haider Munske

Über lexikalischen Schrott

Georg Objartel

Semantische Individualisierung.

Ansätze zu Goethe 
Jost Schillemeit

,Erlebnis‘.

Bemerkungen eines Literarhistorikers zu einer Wortbildung

des 19. Jahrhunderts

\section{Semantik und Pragmatik}

Hans-Joachim Behr

Von Wodan bis Henne?

Überlegungen zur Klassifikation und Pragmatik einiger

althochdeutscher und altsächsischer Zauber- und Segensprüche

Helmut Rehbock

Gewusst, wem!

Exzitative Nebensätze zwischen Alltag und Literatur

Heikki J. Hakkarainen

Minimalistische grammatische und lexikalische Hinweise zur pragmatischen Positionierung von Hörfunkwerbung

Herbert Blume

Katt och råtta, Katz und Maus.

Ungleiche lexikalisch-semantische Strukturen im Schwedischen

und Deutschen

Els Oksaar

Pragmatische Aspekte des Schweigens.

Interkulturelle Betrachtungen

Wenliang Yang

„Drache“ oder ,Tiger“?

Interkulturelle Interferenzen Chinesisch-Deutsch

\section{Wissenschaftsgeschichte der Germanistik}

Dieter Cherubim

Pathologia linguae.

Die „Krankheiten“ der Sprache und deren Remedur 
VIII

Armin Burkhardt

Jacob Grimm als Politiker ........................................... 449

Karl Stackmann

Edward Schröder an Hermann Paul.

Ein Brief ,aus Feindesland“

7. Anhang

Ehren=Dialog

Verzeichnis der Schriften von Helmut Henne

493

Verzeichnis der von Helmut Henne betreuten

Dissertationen und Habilitationen

8. Tabula gratulatoria 